\title{
In silico studies of conformational changes in wheat heat shock proteins
}

\begin{abstract}
One of the key limiting factors for life on the planet Earth is temperature. Therefore, nearly all the organisms have developed some biosignaling system to feel thermal changes in the environment and regulate their metabolic process to avoid any loss due to an increased temperature. Heat stress is among the most important environmental challenges against the crop plants with an adverse effect on plant growth, development reproduction, and yield. The physiological response towards high temperature (i.e. heat stress) leads to the altered proteomic profile of wheat plant resulting in increased level of heat shock proteins (HSPs). The rate of HSPs synthesis is directly correlated with the rising temperature. The HSPs constitute a family of highly conserved proteins. They sample large conformational changes under the effect of elevated temperature. In this opinion paper, conformational analysis of heat shock proteins is suggested to understand their functioning. The computational methods for studying bio molecular 3D structures and conformations provide an opportunity to empathize their function.
\end{abstract}

Volume 5 Issue I - 2017

\author{
Surya Pratap Singh,Vinay Sharma \\ Department of Bioscience and Biotechnology, Banasthali \\ University, India
}

\begin{abstract}
Correspondence: Department of Bioscience and Biotechnology, Banasthali University, Banasthali 304022 Rajasthan, India, Email vinaysharma30@yahoo.co.uk
\end{abstract}

Received: December 31, 2016 | Published: February 02, 2017

Keywords: heat stress, heat shock proteins, molecular modeling, conformational analysis

\section{Opinion}

Wheat (Triticum aestivum) is one of the most important staple food crops, cultivated worldwide. The increased human population would require a major augment in the food supply in coming decades. Extension of sowing fields has only a little possibility; therefore, an increase the crop productivity has become a crucial necessity to assure the future food security. ${ }^{1,2}$ Increased use of fertilizers and improved pest management has led to a significant increase in the gross wheat production, ${ }^{3}$ however, an expectation of increase in mean temperature by 1.8 to $5.8^{\circ} \mathrm{C}$ as well frequent hot days increase owing to the global climate changes; moreover, Luzuriaga et al., ${ }^{4}$ Hedhly et al., ${ }^{5}$ and Huang et al., ${ }^{6}$ have also reported the effect of elevated temperature, during grain filling, over the crop. Drought and high light conditions which are also known to damage crops $^{7-9}$ generally accompany elevated temperature Plants being pedunculate are unable to escape heat thus are required to modify their metabolism by utilizing valuable resources to avoid any damage, through acclimation. Otherwise, the programmed cell death is activated in specific cells or tissues leading to leaf abscission, terminating flowering and fruiting or death of the whole plant. During the period of 1980-2008the heat stress has resulted in reduced production of wheat and maize by $5.5 \%$ and $3.8 \%$ respectively. ${ }^{10}$ Therefore, it becomes extremely important to understand the temperature sensitivity of crop plants.

The optimal temperature during reproductive (i.e. anthesis and grain filling) stage of wheat varies between $12-22^{\circ} \mathrm{C} . .^{11,12}$ The temperature elevation above the optimal (i.e. heat stress) in terms of magnitude and the exposure duration has catastrophic events over the morphology and physiology of the plants. ${ }^{13}$ Wheat is highly sensitive to elevated temperature as well as increased exposure to the high temperature at different degrees; however, the heat stress during reproductive phase has disastrous effects leading to declined quality of grains (i.e. reduced dry weight) and the overall yield. The late sown wheat is highly susceptible to heat stress during the reproductive period leading to a substantial fall in yield. Therefore the elevated temperature during grain filling period (i.e. terminal heat stress) is a challenging problem for wheat cultivation in forthcoming decades. ${ }^{14}$

\section{The heat shock proteins}

Heat stress, an abiotic stress, is among the best studied environmental responses in wheat and other crop plants. The heat stress adversely affects the stability of different proteins, membrane fluidity cytoskeleton and enzyme kinetics resulting in a metabolic imbalance in the plant. ${ }^{15}$ These physiological responses towards high temperature lead to the altered proteomic as well as the transcriptomic profile of plants, which is a similar event in other organisms. Among the plants, thermotolerance is achieved by different methods such as synthesis of isoprene, glycinebetaine or antioxidant enzymes and the heat shock proteins (HSPs). ${ }^{16}$ Wheat like many other crop plants adopts the synthesis of HSPs in order of thermotolerance. The level of HSPs in wheat plants increases as a consequence of heat stress, leading to an altered proteome. The rate of HSPs synthesis is synchronized with temperature, and the temperature beyond optimal results in the maximum HSPs synthesized. ${ }^{17,18}$ This synchronization is suggestive of the physiological response of an organism towards the elevated temperature. HSPs constitute a family of highly conserved proteins among the eukaryotes, suggesting that the synthesis of HSPs is an essential event under the heat stress.

HSPs are the molecular chaperones, the key role of these proteins is in protein folding, assembly and translocation of various proteins in normal cellular process moreover they are also responsible for stabilizing proteins and membranes and the refolding of proteins under stress. The HSPs are grouped into five major classes: the Hsp100 (Clp) family; the Hsp90 family; the Hsp70 (DnaK) family; the chaperonins (GroEL and Hsp60); and the small Hsp (sHsp) family. The molecular localization of the HSPs is in both cytoplasm and organelles such as Chloroplasts, mitochondria, and endoplasmic reticulum. These HSPs have different target proteins, binding noncovalently they do not form any final product the Hsp60 and Hsp70 are widely studied HSPs. ${ }^{19}$ 


\section{Computational methods}

Study of protein three-dimensional structures is crucial for understanding their functioning. The powerful experimental method such as x-ray crystallography and NMR are used to determine the 3D structure of proteins, however, both of these methods suffers from shortcomings such as time-consuming, expensive and complications of the experimental setup..$^{20}$ In the recent years in silico methods such as molecular modeling and simulations are widely used in the timely study of protein structures and dynamics.

\section{Modeling 3D structure of HSPs}

The experimental structures of wheat HSPs are not available in protein databank (PDB). Therefore, computational methods can serve as a useful tool for predicting the 3D structure of HSPs in a timely manner. The homology modeling is one of the popular methods used for protein $3 \mathrm{D}$ structure starting from its sequence. The basic concept of this method is homologous (i.e. evolutionarily related) protein share similar 3D structure. ${ }^{21}$ However, in most of the cases alignment is not perfect due to several insertions and deletion events and gaps are introduced at many places during sequence alignment, leading to the erroneous prediction of loop regions, therefore, the computational modeling of protein three-dimensional structures. These loops show reasonably large conformational changes as compared with regular secondary structures. With the development of more sophisticated algorithms and computational power in recent years, a variety of methods has been developed for protein structure prediction. A comprehensive list of protein modeling programs is given in Table 1.

Table I Popular protein 3D structure prediction programs

\begin{tabular}{lll}
\hline Name & Description & Link \\
\hline MODELLER & $\begin{array}{l}\text { Homology-based protein 3D structure prediction program. Free for academic users. } \\
\text { Rosetta homology modeling and } a b \text { initio fragment assembly. Academic registration }\end{array}$ & http://salilab.org/modeller/ \\
ROBETTA & $\begin{array}{l}\text { required for server access. } \\
\text { Physicochemical energy functions based. Commercial package with GUI interface. }\end{array}$ & http://www.schrodinger.com/ \\
SWISS-MODEL & Fragment assembly based freely accessible automated server. & http://swissmodel.expasy.org/ \\
I-TASSER & The combination of threading and ab inito folding method. & http://zhanglab.ccmb.med.umich.edu/l-TASSER/ \\
Bhageerath & Modeling protein structure at the atomic level. & http://www.scfbio-iitd.res.in/bhageerath
\end{tabular}

Molecular dynamics simulation and conformational analysis of HSPs

The biological activity of a protein is derived from its flexibility and undergoes temporal conformational changes. ${ }^{22}$ However, the crystal structures, as well as the computational models of proteins, represent only single frame (i.e. conformation) of the molecule thus they are incapable of explaining the conformational fluctuations and the mechanism of action absolutely. Fortunately, the molecular dynamics simulation has evolved as a powerful technique, based on the information derived from the particles interaction potential, to understand dynamics and conformational changes of proteins. ${ }^{23}$ The temporal molecular flexibility of proteins is estimated in terms of root mean square fluctuations (RMSF) and the dynamical behavior of loops and overall structures are measured. ${ }^{24}$ There are some popular packages used for MD simulation of proteins. A comparative list of MD packages in given in Table 2.

Table 2 Molecular dynamics simulation packages

\begin{tabular}{ll}
\hline Name & Description \\
\hline GROMACS & Freely available package for high performance MD simulation. \\
LAMMPS & Free package suitable for coarse graining system \\
AMBER & Non-free, available for academic users at low cost.Very useful package for bimolecular simulation. \\
CHARMM & Mainly commercially available with Accelrys DS, also available free for developers. \\
Desmond & Commercial package available with Schrodinger suit with excellent GUI.
\end{tabular}

HSPs are the molecular chaperones that participate in preserving the functional conformations of a variety of proteins and thus support the cell survival under heat stress. Under the elevated temperature HSPs go through large conformational changes to sustain the effect of heat, therefore the conformational analysis of the HSPs becomes vitally important to understand their functioning at high temperatures. The conformational analysis of proteins can be performed on MD simulation data. The principal component analyses are used to mine the huge conformational space of proteins. ${ }^{25}$ The potential energy surface (PES) of the proteins represents the hyper surface of its potential energy as a function of coordinates. Molecular dynamics together with essential dynamics offers a sampling of the protein conformations from which the free energy landscapes are generated to assist the conformational analysis ${ }^{26}$ (Figure 1). Singh et al., ${ }^{27}$ has shown MD simulation based conformational studies of $B$. subtilis lipases at different temperatures, similar studies on the modeled wheat HSPs can be performed at different temperatures to understand the effect of heat stress on the HSPs conformations and improvement wheat varieties. 


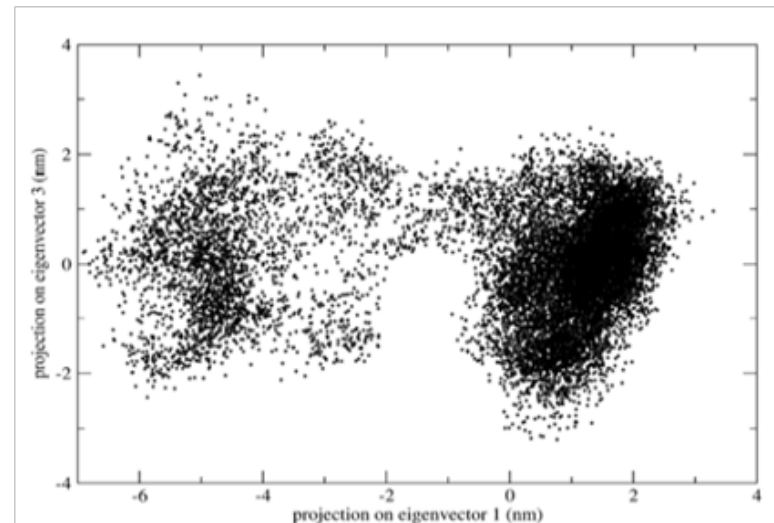

A

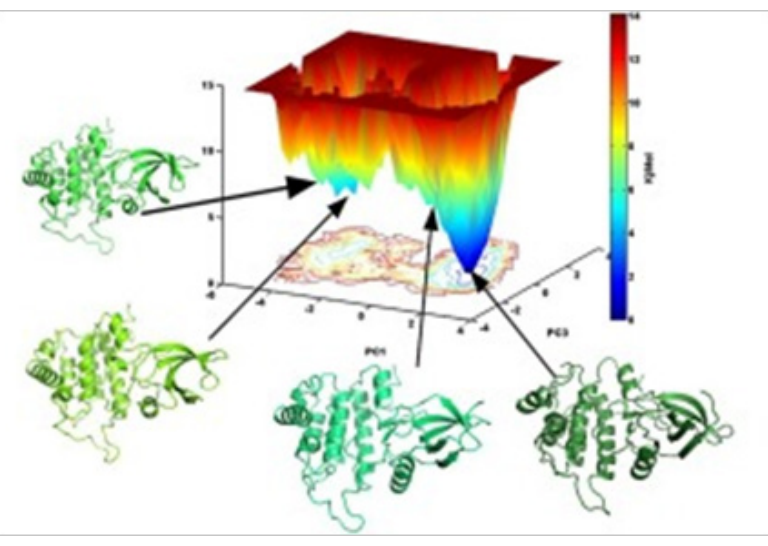

Figure I Conformational changes in protein. (A) Conformational substates in CKI and (B) Free energy landscape analysis of the conformations show conformational changes in the molecule.

\section{Conclusion}

Wheat is staple crop sown worldwide its yield is likely to be affected by the heat stress in upcoming decades. The HSPs are the class of proteins that participate in regulating and maintaining the functional conformation of a variety of other proteins for cell survival, however, they also undergo large conformational changes at elevated temperatures to maintain their function. The computational based conformational analyses are reliable methods to understand the functioning of proteins.

\section{Acknowledgements}

The authors are thankful to the Department of Biotechnology; Govt. of India supported Centre for Bioinformatics at Banasthali University for the extensive use of computational facilities and literature survey work.

\section{Conflict of interest}

The author declares no conflict of interest.

\section{References}

1. Parry MAJ, Reynolds MP, Salvucci ME, et al. Raising yield potential of wheat. II. Increasing photosynthetic capacity and efficiency. $J$ Exp Bot. 2011;62(2):453-467

2. Reynolds MP, Bonnett D, Chapman SC, et al. Raising yield potential of wheat. I. Overview of a consortium approach and breeding strategies. $J$ Exp Bot. 2011;62(2):439-452.

3. Semenov MA, Mitchell RAC, Whitmore AP, et al. Shortcomings in wheat yield predictions. Nature Climate Change. 2012;2:380-382.

4. Luzuriaga, AL, Escudero A, Pérez-García F. Environmental maternal effects on seed morphology and germination in Sinapis arvensis (Cruciferae). Weed Res. 2006;46(2):163-174.

5. Hedhly A, Hormaza JI, Herrero M. Global warming and sexual plant reproduction. Trends Plant Sci. 2009;14(1):30-36.

6. Huang Z, Foottit S, Finch-Savage WE. The effect of temperature on reproduction in the summer and winter annual Arabidopsis thaliana ecotypes Bur and Cvi. Ann Bot. 2014;113(6):921-929.

7. Mittal S, Kumari N, Sharma V. Differential responses of seven contrasting species to high light using pigment and chlorophyll a fluorescence. $J$ Stress Physiology and Biochemistry. 2011;7(2):20-33.
8. Sharma V, Sekhwal MK, Swami AK, et al. Identification of drought responsive proteins using gene ontology hierarchy. Bioinformation. 2012;8(13):595-599.

9. Mittler R, Finka A, Goloubinoff P. How do plants feel the heat? Trends in Biochemical Sciences. 2012;37(3):118-125.

10. Batra NG, Kumari N, Sharma V. Drought-induced changes in chlorophyll fluorescence, photosynthetic pigments and thylakoid membrane proteins of Vigna radiata. J Plant Interactions. 2014;9(1):712-721.

11. Lobell, DB, Schlenker W, Roberts JC. Climate trends and global crop production since 1980. Science. 2011;333(6042):616-620.

12. Zhao H, Dai TB, Jing Q, et al. Leaf senescence and grain filling affected by post-anthesis high temperatures in two different wheat cultivars. Plant Growth Regul. 2007;51(2):149-158.

13. Zhao H, Dai T, Jiang D, et al. Effects of high temperature on key enzymes involved in starch and protein formation in grains of two wheat 1320 cultivars. J Agron Crop Sci. 2008;194:47-54.

14. Wahid A, Gelani S, Ashraf M, et al. Heat tolerance in 1260 plants: an overview. Environ Exp Bot. 2007;61(2007):199-223.

15. Mitra R, Bhatia CR. Bioenergetic cost of heat tolerance in wheat crop. Curr Sci. 2008;94:1049-1053.

16. Young LW, Wilen RW, Bonham-Smith PC. High temperature stress of Brassica napus during Flowering reduces micro- and megagametophyte fertility induces fruit abortion, and disrupts seed production. $J$ Exp Bot. 2004;55(396):48 - 495 .

17. Vierling E. The roles of heat shock proteins in plants. Annu Rev Plant Physiology Plant Mol Biology. 1991;42:579-620.

18. Jacob P, Hirt H, BendahmaneA. The heat shock protein/chaperone network and multiple stress resistance. Plant Biotechnol J. 2017;15(4):405-414.

19. Wang W, Vinocur B, Shoseyov O, et al. Role of plant heat-shock proteins and molecular chaperones in the abiotic stress response. Trends Plant Sci. 2004;9(5):244-252.

20. Wang JF, Wei DQ, Lin Y, et al. Insights from modeling the 3D structure of NAD (P) H-dependent D-xylose reductase of Pichia stipitis and its binding interactions with NAD and NADP. Biochem Biophys Res Commun. 2007;359(2):323-329.

21. Sali A, Blundell TL. Comparative modelling by satisfaction of spatial restraints. J Mol Biol. 1993;234(3):779-815.

22. Henzler-Wildman K, Kern D. Dynamic personalities of proteins. Nature. 2007;450(7172):964-972. 
23. Karplus M, McCammon JA. Molecular dynamics simulations of biomolecules. Nat Struct Biol. 2002;9(9):646-652.

24. Singh SP, Gupta DK. A comparative study of structural and conformational properties of casein kinase-1 isoforms: Insights from molecular dynamics and principal component analysis. J Theo Biol. 2015;371:59-68.

25. Amadei A, Linssen ABM, Berendsen HJC. Essential dynamics of proteins. Proteins. 1993;17(4):412-425.
26. Singh SP, Gupta DK. Dynamic conformational ensembles regulate casein kinase-1 isoforms: Insights from molecular dynamics and molecular docking studies. Comp Biol Chem. 2016;61:39-46.

27. Singh B, Bulusu G, Mitra A. Understanding the thermostability and activity of Bacillus subtilis lipase mutants: Insights from molecular dynamics simulations. J Phys Chem B. 2015;119(2):392-409. 